\title{
A model study on changes of European and Swiss particulate matter, ozone and nitrogen deposition between 1990 and 2020 due to the revised Gothenburg protocol
}

\author{
S. Aksoyoglu et al.
}

Correspondence to: S. Aksoyoglu (sebnem.aksoyoglu@psi.ch) 


\section{Supplementary material:}

3 Figure S1: Time series of measured (24 ANETZ stations) and modelled (WRF) specific 4 humidity $\left(\mathrm{kg} \mathrm{kg}^{-1}\right)$, air temperature $(\operatorname{deg} \mathrm{C})$ and wind speed $\left(\mathrm{m} \mathrm{s}^{-1}\right)$. MBE: mean bias 5 error, r: correlation coefficient.

6 Figure S2: Annual average $\mathrm{PM}_{2.5}\left(\mu \mathrm{g} \mathrm{m}^{-3}\right)$ over the European (upper panel) and Swiss 7 (lower panel) domains for the reference case (2005).

8 Figure S3: Observed yearly average PM10 values, raw (blue) and adjusted for 9 meteorology (red), the respective trend lines obtained by linear regression (from 10 Barmpadimos et al. 2011). The modelled annual average PM10 values from this study 11 for 1990, 2005 and 2006 are shown with black crosses.

12 Figure S4: Annual average $\mathrm{O}_{3}$ mixing ratios (ppb) over the European (upper panel) and 13 Swiss (lower panel) domains for the reference case (2005).

14 Figure S5: Relative changes in AOT40 over the European domain, BL 2020- 2005 15 (upper panel) and MTFR 2020- 2005 (lower panel).

16 Figure S6: Relative changes in AOT40 over the Swiss domain, BL 2020 - 2005 (upper 17 panel) and MTFR 2020- 2005 (lower panel).

18 Figure S7: Relative changes in SOMO35 over the European domain for two scenarios:

19 BL 2020- 2005 (upper panel) and MTFR 2020 - 2005 (lower panel).

20 Figure S8: Relative changes in SOMO35 over the Swiss domain for two scenarios: BL 21 2020- 2005 (upper panel) and MTFR 2020 - 2005 (lower panel).

22 Figure S9: Dry (upper panel) and wet (lower panel) nitrogen deposition (kg N ha-1 $\mathrm{y}^{-1}$ ) 23 over the European domain (2006). 

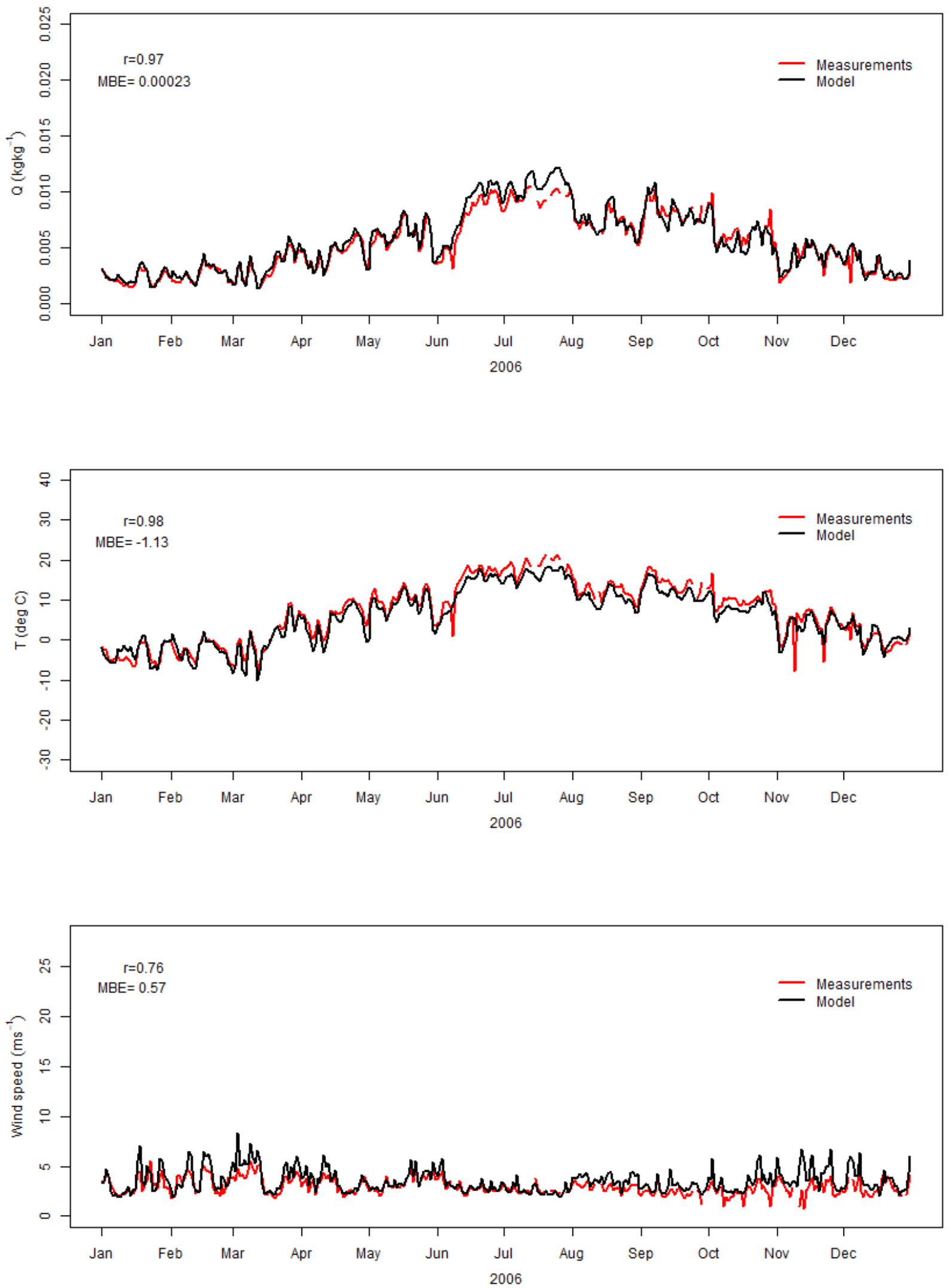

5 Figure S1: Time series of measured (24 ANETZ stations) and modelled (WRF) specific 6 humidity $\left(\mathrm{kg} \mathrm{kg}^{-1}\right)$, air temperature $(\operatorname{deg~} \mathrm{C})$ and wind speed $\left(\mathrm{m} \mathrm{s}^{-1}\right)$. MBE: mean bias 7 error, r: correlation coefficient. 

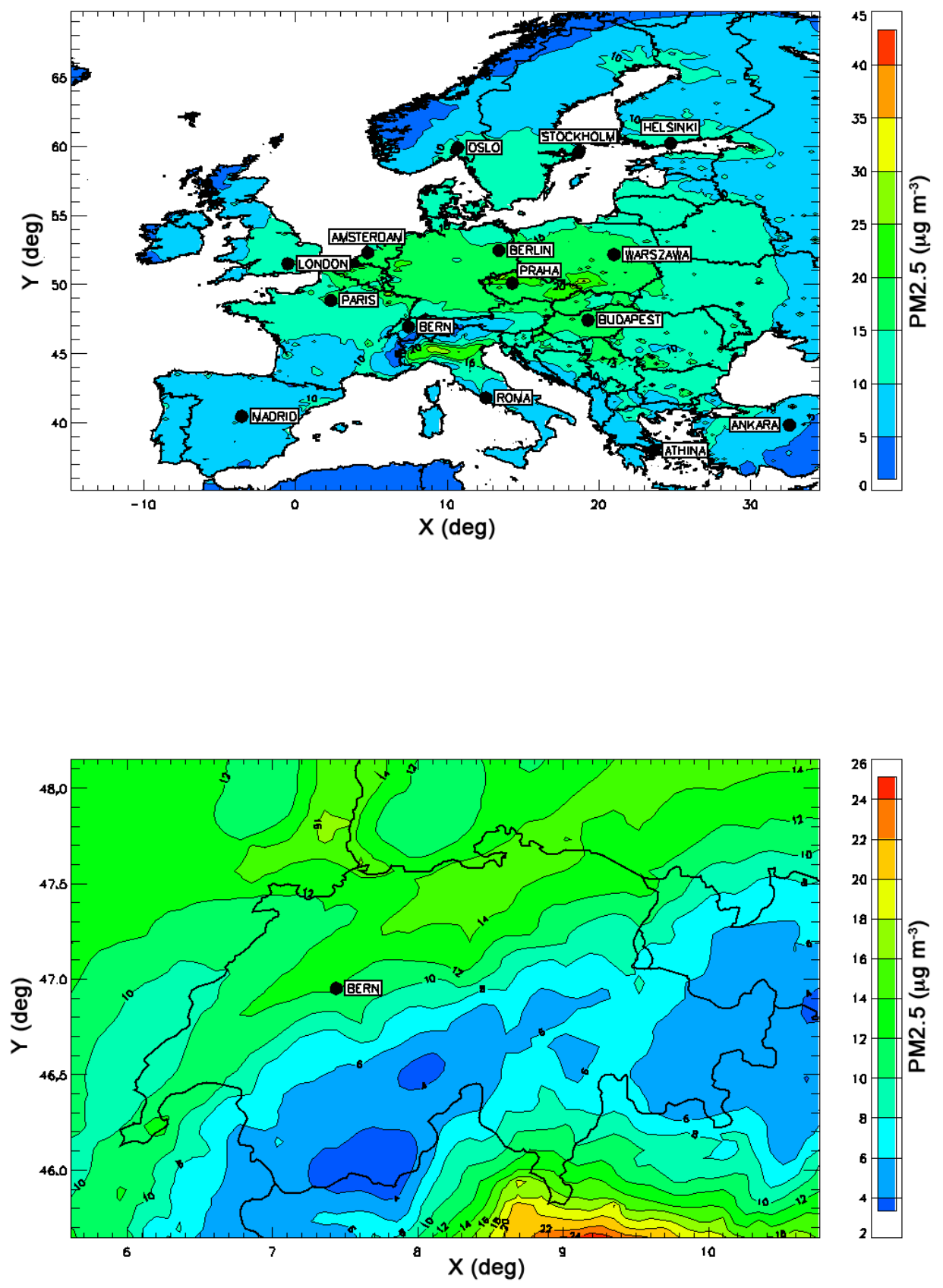

4 Figure S2: Annual average $\mathrm{PM}_{2.5}\left(\mu \mathrm{g} \mathrm{m}^{-3}\right)$ over the European (upper panel) and Swiss 5 (lower panel) domains for the reference case (2005). 

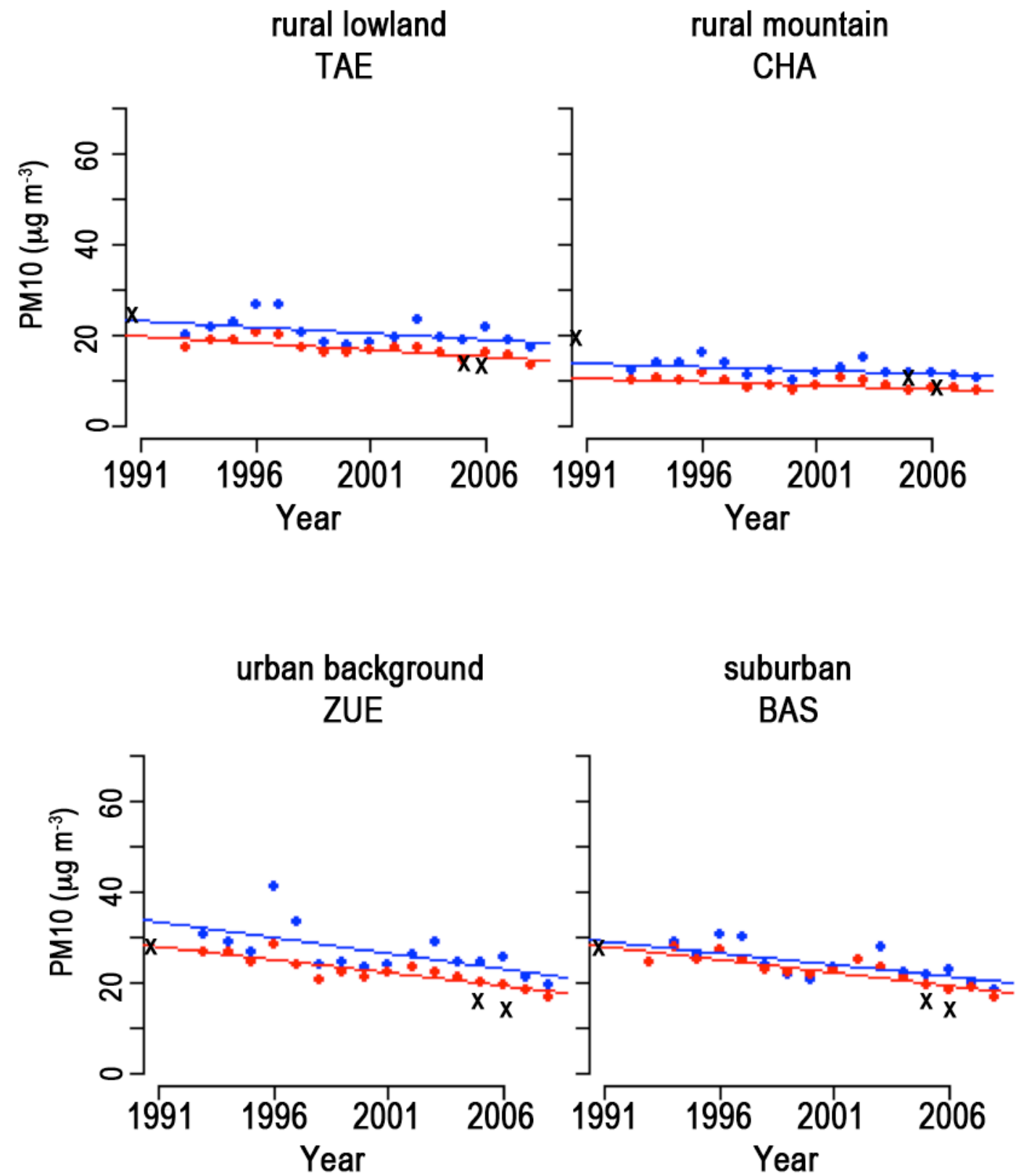

4 Figure S3: Observed yearly average PM10 values at four stations (TAE: rural, lowland, 5 CHA: rural, mountain, ZUE: urban background, BAS: suburban), raw (blue) and adjusted 6 for meteorology (red), the respective trend lines obtained by linear regression (from 7 Barmpadimos et al. 2011). The modelled annual average PM10 values from this study 8 for 1990, 2005 and 2006 are shown with black crosses. 

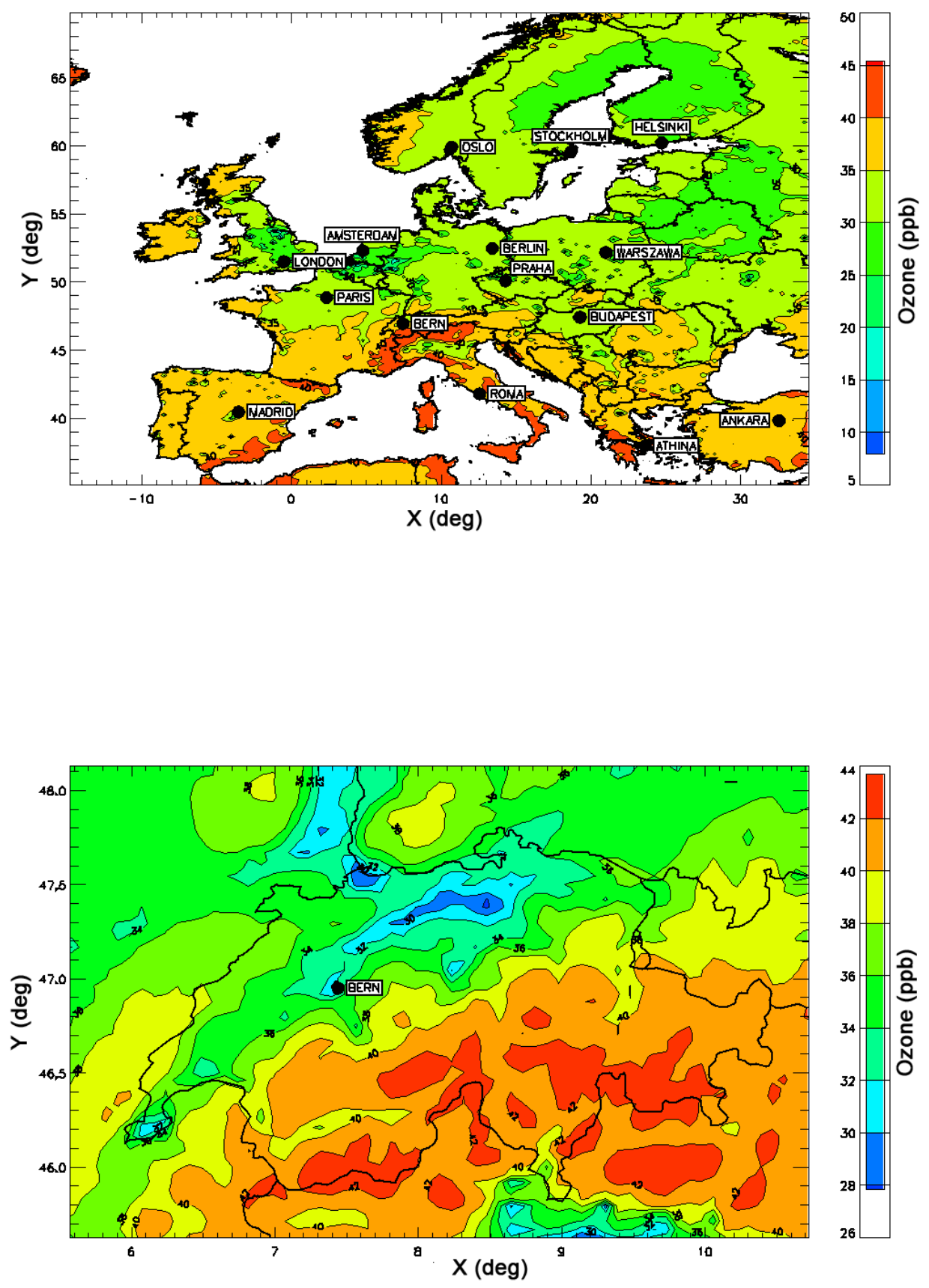

3 Figure S4: Annual average $\mathrm{O}_{3}$ mixing ratios (ppb) over the European (upper panel) and 4 Swiss (lower panel) domains for the reference case (2005). 

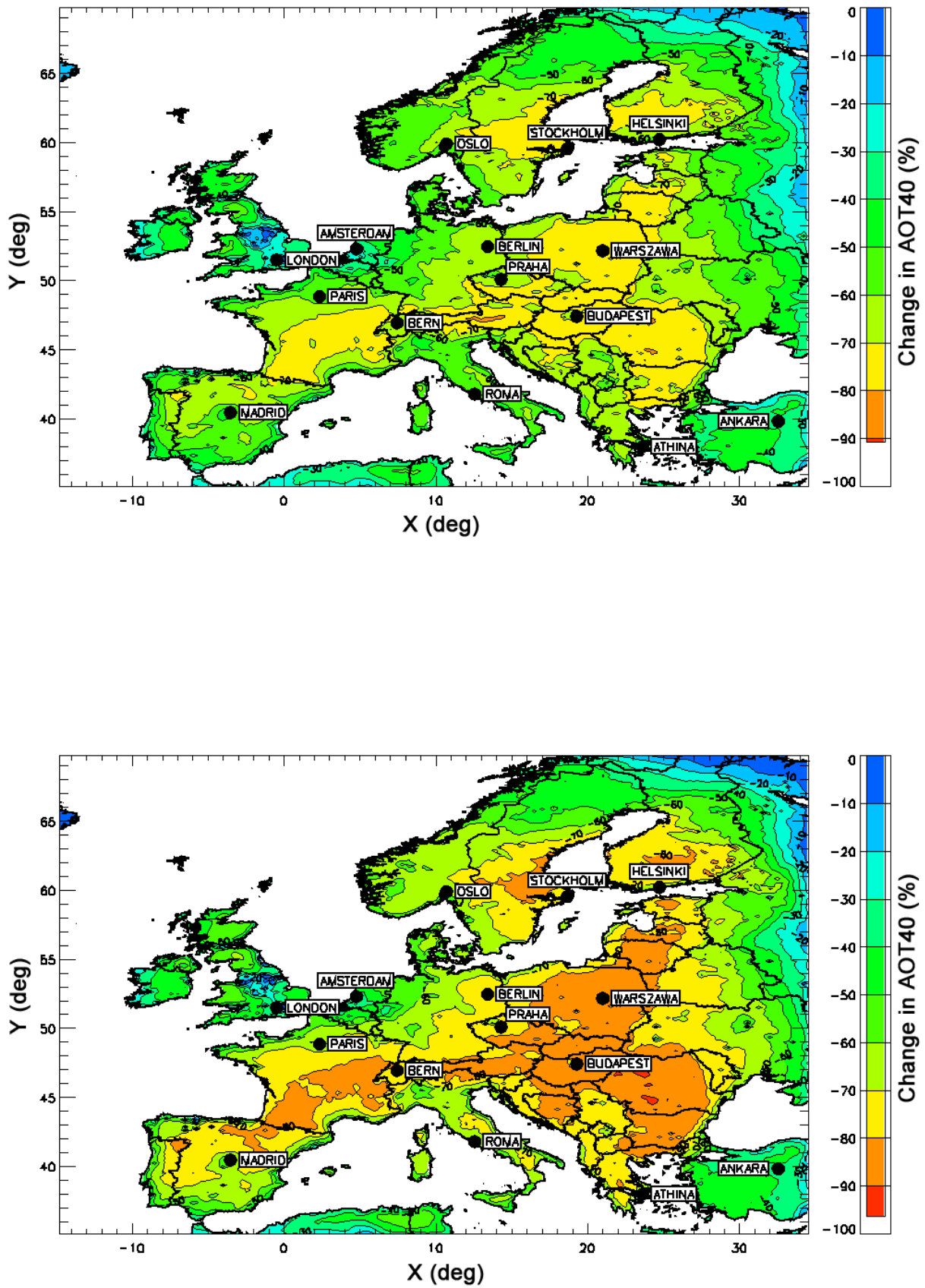

2

3 Figure S5: Relative changes in AOT40 over the European domain, BL 2020- 2005 4 (upper panel) and MTFR 2020- 2005 (lower panel). 

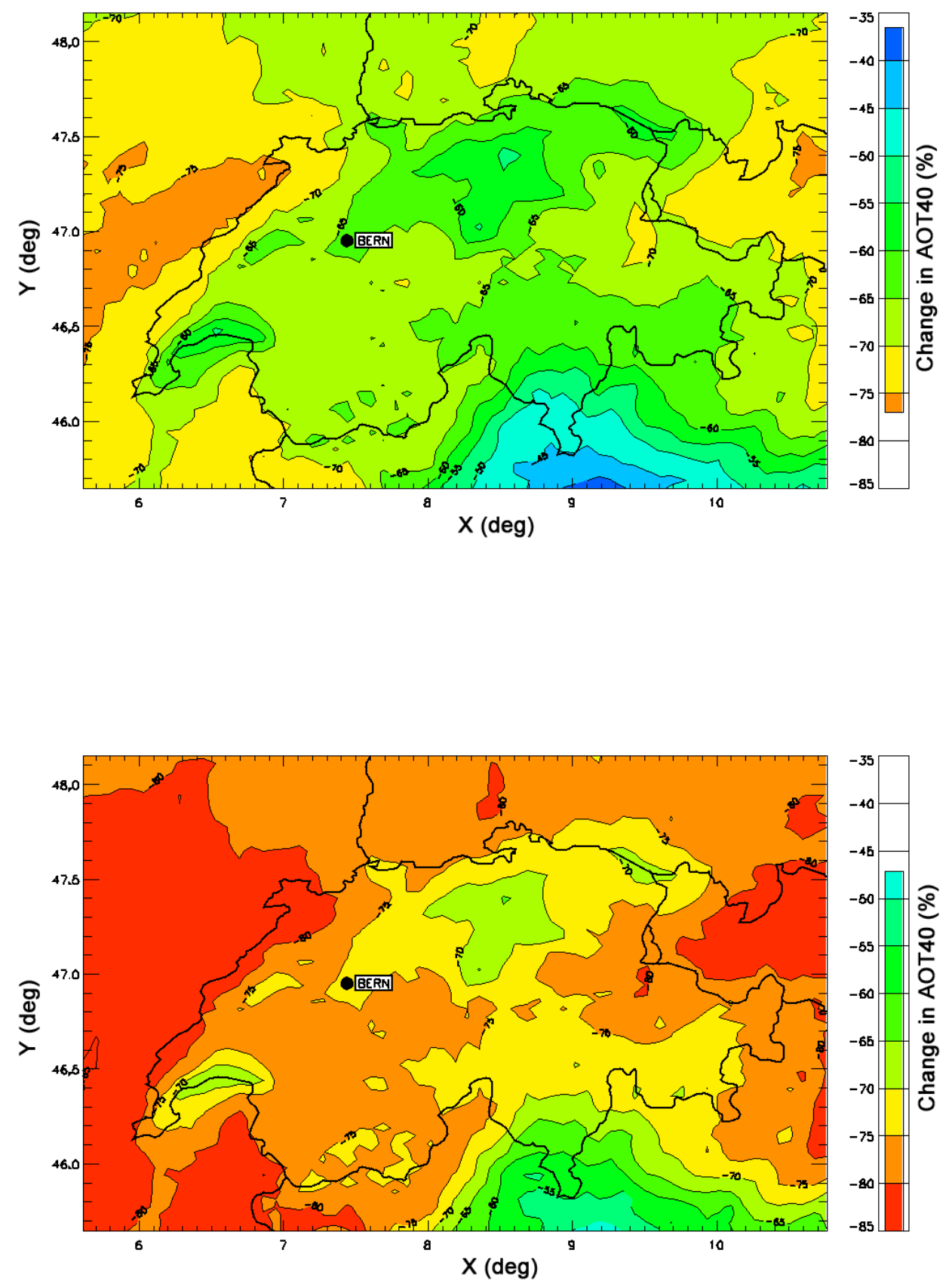

3 Figure S6: Relative changes in AOT40 over the Swiss domain, BL 2020 - 2005 (upper 4 panel) and MTFR 2020- 2005 (lower panel). 

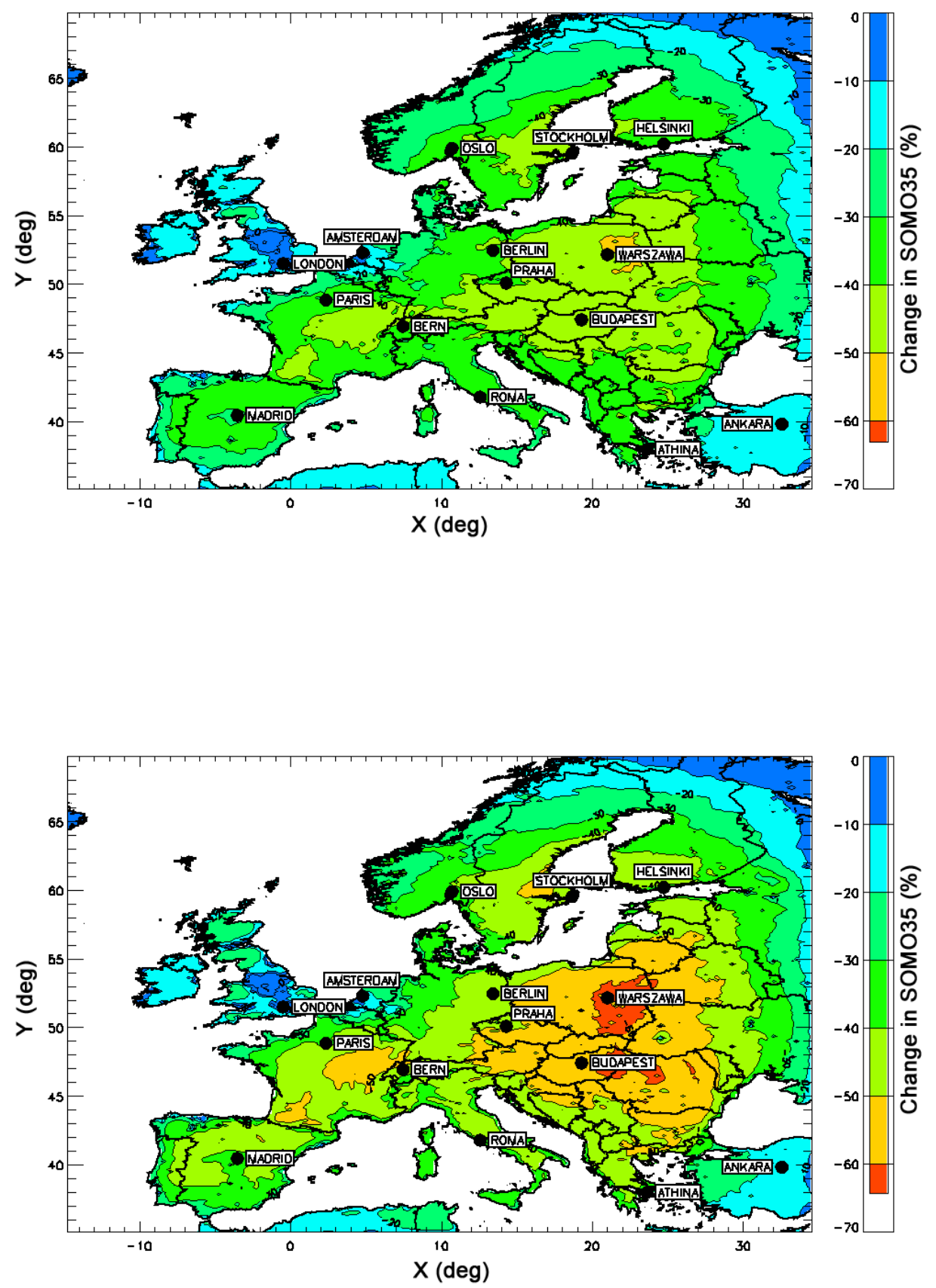

4 Figure S7: Relative changes in SOMO35 over the European domain for two scenarios:

5 BL 2020- 2005 (upper panel) and MTFR 2020 - 2005 (lower panel). 

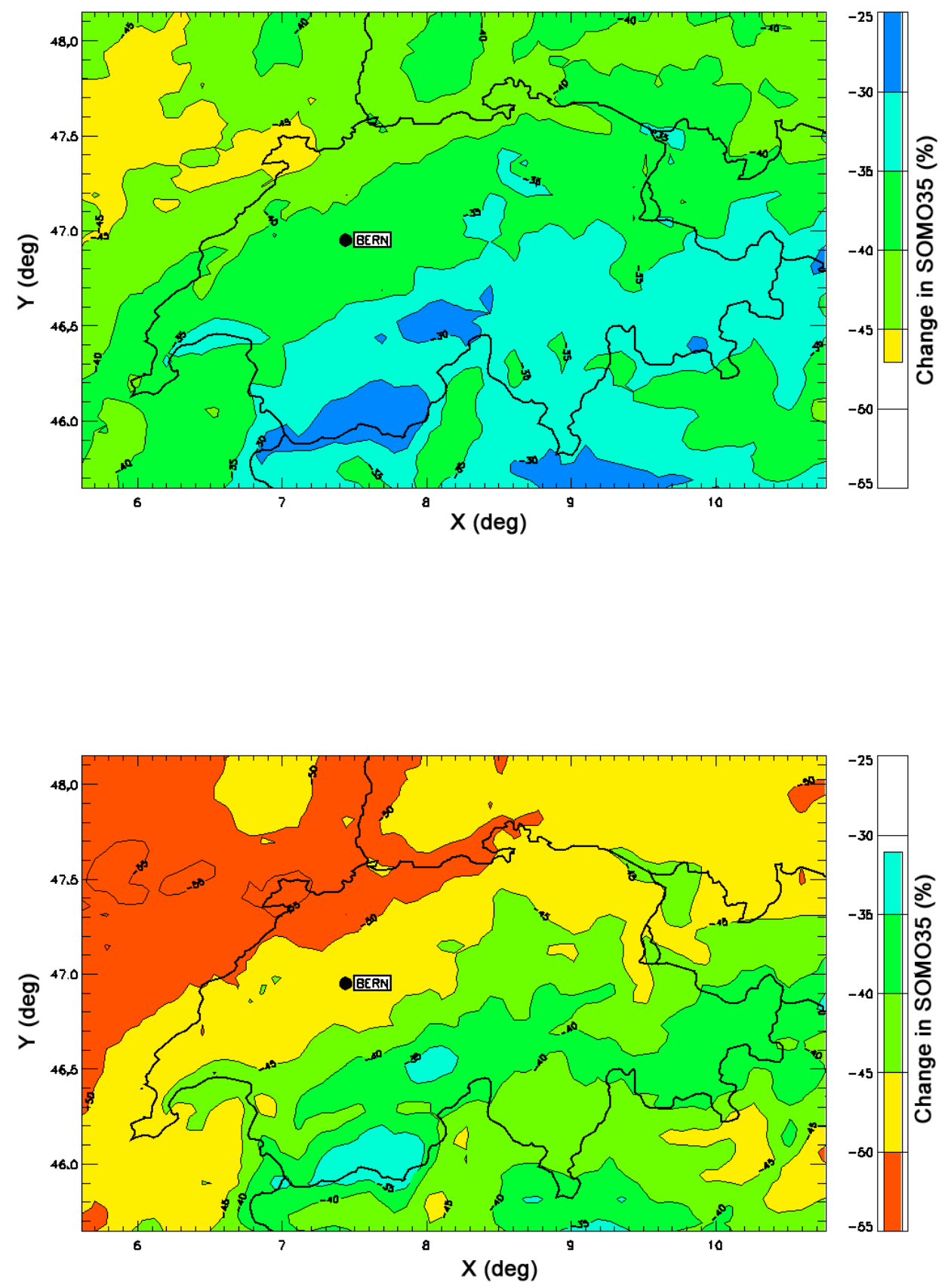

2

3 Figure S8: Relative changes in SOMO35 over the Swiss domain for two scenarios: BL 4 2020- 2005 (upper panel) and MTFR 2020 - 2005 (lower panel).

5

6

7

8 

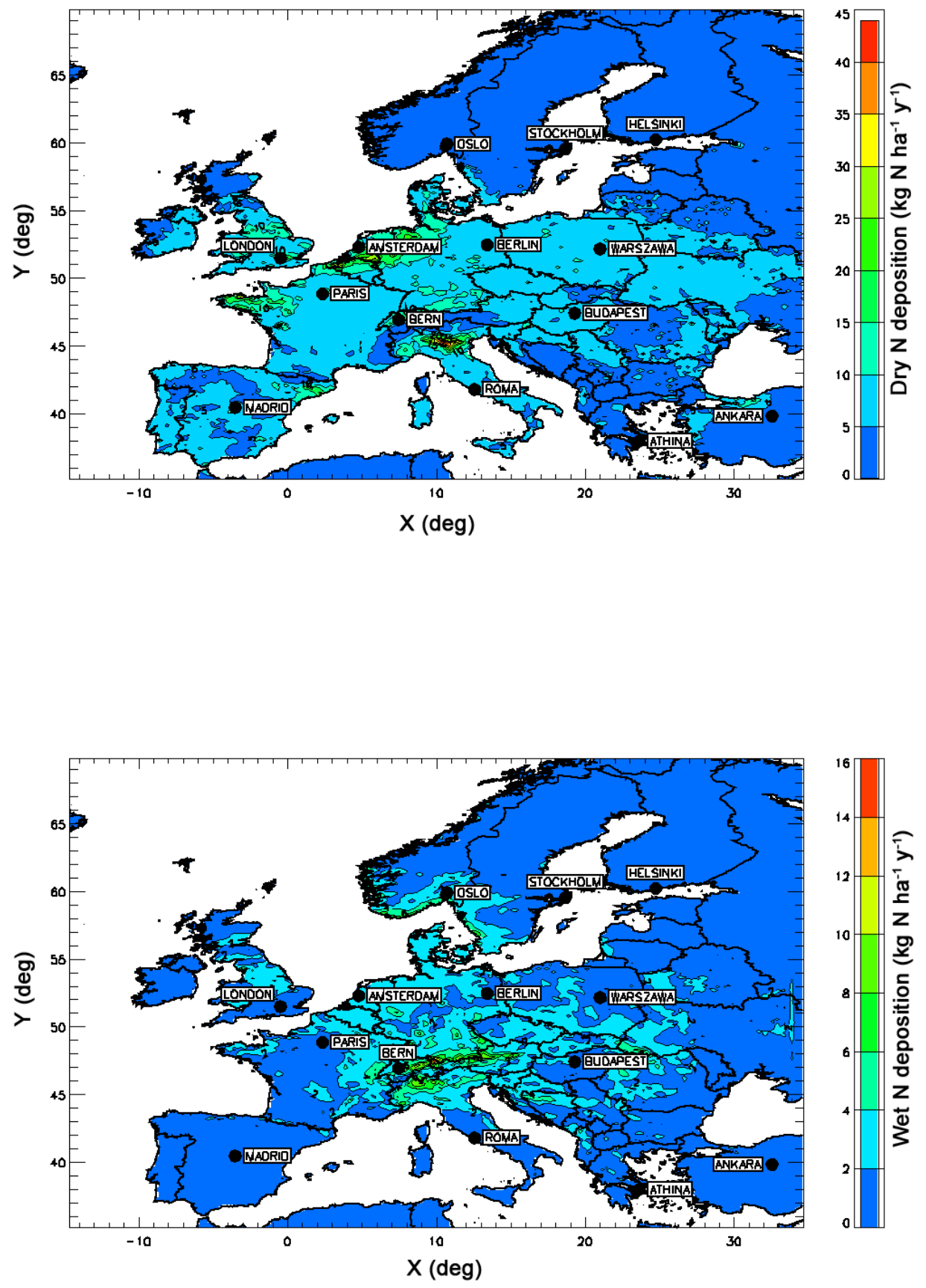

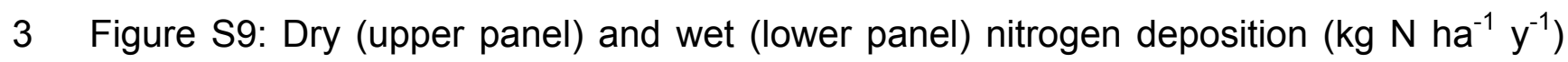
4 over the European domain (2006). 